\title{
Figure S6
}

\section{Diversity of ScTIR1 genomic copies.}

Full length genomic copies of ScTIR1 were retrieved from the genome using REPET and aligned (clustalW). The aligment was used to construct a phylogenetic tree using Maximum parsimony. Copies with identical sequences are encircled.

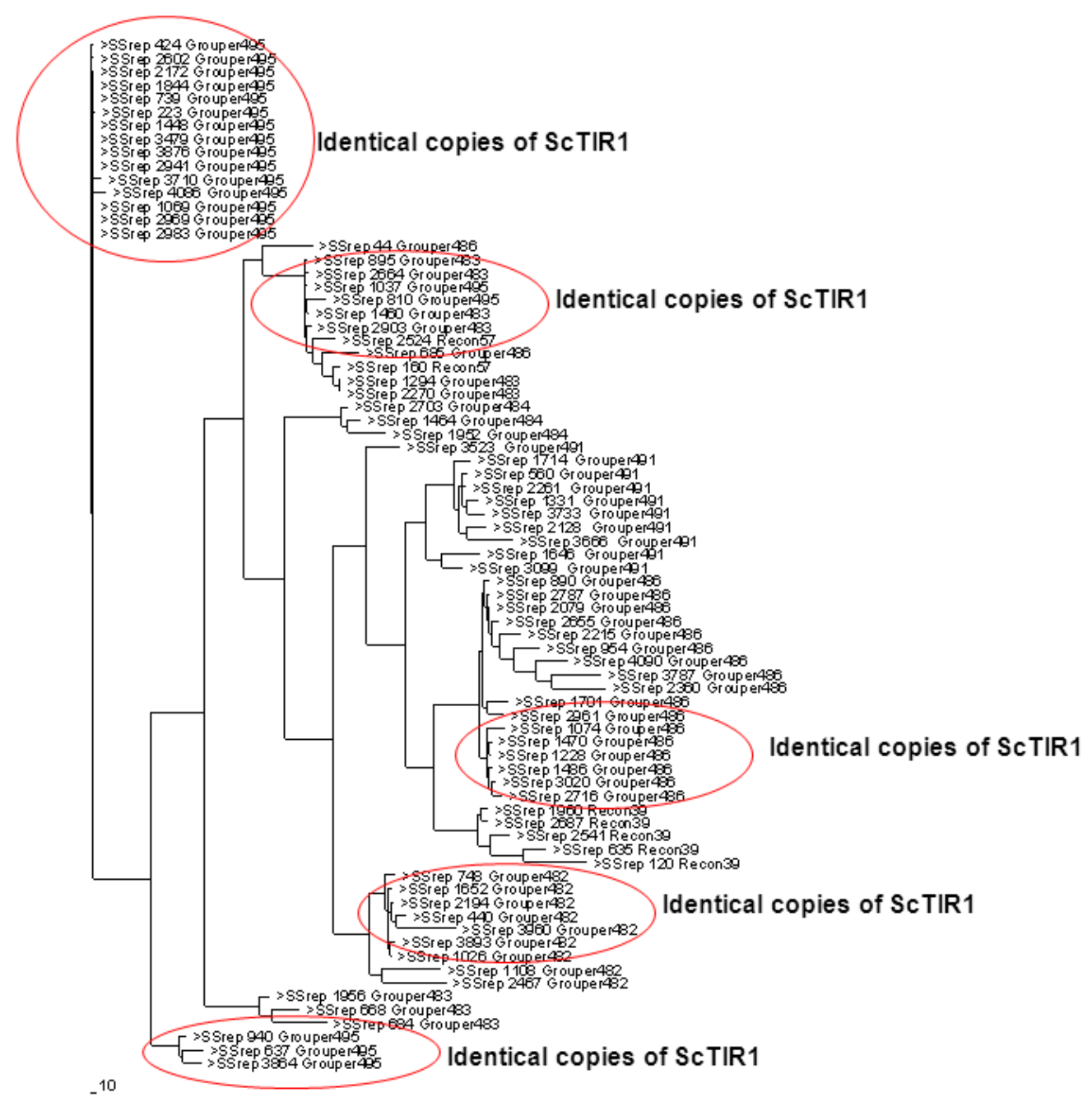

